\title{
Beneficial Effects of a Dietary Weight Loss Intervention on Human Gut Microbiome Diversity and Metabolism Are Not Sustained during Weight Maintenance
}

\author{
Femke-Anouska Heinsen $^{a} \quad$ Daniela Fangmann ${ }^{b}$ Nike Müller ${ }^{b}$ \\ Dominik M. Schulte ${ }^{b} \quad$ Malte C. Rühlemann ${ }^{a}$ Kathrin Türk ${ }^{b}$ Ute Settgast ${ }^{b}$ \\ Wolfgang Lieb ${ }^{c}$ John F. Baines ${ }^{d}$ Stefan Schreiber ${ }^{a, b}$ Andre Franke ${ }^{a}$ \\ Matthias Laudes ${ }^{b}$ \\ ${ }^{a}$ Institute of Clinical Molecular Biology, University of Kiel, Kiel, Germany, ${ }^{b}$ Department of \\ Internal Medicine 1, University of Kiel, Kiel, Germany, ' Institute for Epidemiology, University \\ of Kiel, Kiel, Germany, d Max Planck Institute for Evolutionary Biology, Plön, Germany
}

\section{Key Words}

Obesity $\cdot$ Microbiome $\cdot$ Weight maintenance

\begin{abstract}
Objective: In the present study, we examined the effect of a very low-calorie $\operatorname{diet}(\mathrm{VLCD})$ based obesity program on human gut microbiome diversity and metabolism during weight loss and weight maintenance. Methods: Obese subjects underwent 3 months of VLCD followed by 3 months of weight maintenance. A lean and an obese control group were included. The microbiome was characterized by performing high-throughput dual-indexed 16S rDNA amplicon sequencing. Results: At baseline, a significant difference in the Firmicutes/Bacteroidetes ratio between the lean and obese individuals was observed ( $p=0.047)$. The VLCD resulted in significant alterations in gut microbiome diversity from baseline to 3 months $(p=$ 0.0053). Acinetobacter represented an indicator species for the observed effect (indicator value $=0.998, p=0.006$ ). Metabolic analyses revealed alterations of the bacterial riboflavin pathway from baseline to 3 months $\left(p_{n o m}=0.0078\right)$. These changes in diversity and bacterial metabolism induced by VLCD diminished during the weight maintenance phase, despite sustained reductions in body weight and sustained improvements of insulin sensitivity. Conclusion: The present data show that a VLCD is able to beneficially alter both gut microbiome
\end{abstract}

Femke-Anouska Heinsen and Daniela Fangmann contributed equally to this paper. 
Heinsen et al.: Beneficial Effects of a Dietary Weight Loss Intervention on Human Gut

diversity and metabolism in obese humans, but that these changes are not sustained during weight maintenance. This finding might suggest that the microbiome should be targeted during obesity programs.

(C) 2016 The Author(s)

Published by S. Karger GmbH, Freiburg

\section{Introduction}

Obesity is caused by an imbalance of energy intake and energy expenditure, and it is showing an increasing prevalence. Between 1980 and 2014, obesity has doubled worldwide [1]. Given this epidemic increase, it is important to identify the factors regulating the balance between energy intake and expenditure. In particular, there has been much interest in the gut microbiome recently. The gut microbiome is a complex ecosystem that consists of an immense number of microorganisms and is composed mainly of bacteria but also includes archaea, viruses, fungi, and protozoa [2]. The collective genomes of the microbiota, the bacterial fraction of the microbiome, significantly expanded the host's genetic and metabolic potential and thus complements the host's physiology [2].

It has been shown in a mouse model that colonization of germ-free adult mice with the distal gut microbiota of conventionally raised mice led to an increase in body fat content $(60 \%)$, hepatic production of triglycerides, and insulin resistance within 14 days despite a reduced food intake [3]. There are two major concepts in the association of the gut microbiome and the human energy homeostasis: the 'energy harvest theory' and the 'inflammation theory'. The 'energy harvest theory' implies that the gut microbiome of obese subjects has an increased capacity to harvest energy from the diet by processing otherwise indigestible components of the food [4]. For instance, the microbiota ferments the complex dietary polysaccharides to short-chain fatty acids and monosaccharides, which are then absorbed via the colonic mucosa. Moreover, the deposition of lipids in adipocytes is influenced by the microbiota [3]. The 'inflammatory theory' assumes an activation of the innate immune system in the gut through a specific composition of the microbiota, resulting in a low-grade systemic inflammation characterized by, e.g., an accumulation of macrophages in white adipose tissue [5]. Taken together, these findings suggest that the composition of the gut microbiota plays an important role in energy balance and host metabolism.

Studies of genetically obese (ob/ob) mice revealed that the two major bacterial divisions (phyla) of the gut microbiota, the Firmicutes and the Bacteroidetes, differ between obese and lean mice. The obese animals show a reduction in the amount of Bacteroidetes and an increase in Firmicutes [6]. Aside from that, a linkage between diet and gut microbial ecology in mice has been shown [7]. In contrast to mouse models, there have been conflicting findings in humans regarding the Firmicutes/Bacteroidetes ratio of obese versus lean subjects [8]. While some human studies also identified an increased number of Firmicutes in obesity [9], several other reports did not show significant differences between the proportions of Firmicutes and Bacteroidetes in lean and obese individuals $[10,11]$.

Besides the conflicting findings regarding the Firmicutes/Bacteroidetes ratio, however, there is strong evidence that the gut microbiome also affects energy metabolism in humans:

- It has been shown that the nutritional load influences the overall microbial community in the gut [9] and that a healthier dietary pattern was associated with greater gut microbiome richness [12].

- The transfer of intestinal microbiota from lean donors to patients with metabolic syndrome resulted in improved insulin sensitivity of the recipients [13]. 
Heinsen et al.: Beneficial Effects of a Dietary Weight Loss Intervention on Human Gut

- Two recent human studies reported an overall reduction in gut microbial gene richness associated with human obesity $[14,15]$ and showed an association of the microbial gene counts with the weight loss effects of a nutritional intervention [15].

The aim of this study was to examine i) if a structured, multimodal obesity therapy program including a 3-month formula-based very low-calorie diet (VLCD) (approximately $800 \mathrm{kcal} /$ day) [16] significantly altered the gut microbiota diversity and/or metabolism, and ii) if these potentially beneficial changes in the gut microbiota were sustained during an additional 3-month weight maintenance phase.

\section{Patients and Methods}

\section{Study Participants}

Dietary Intervention Study Population

18 obese human subjects ( 3 men, 15 women) were included into the dietary intervention group. Dietary intervention included a VLCD for 3 months and a subsequent weight maintenance phase of further 3 months. The VLCD consisted of approximately $800 \mathrm{kcal} /$ day, was formula-based and balanced according to micronutrients. The protein amount in the formula meals and thus the proportional intake of protein according to total daily energy intake was elevated (approximately 33\%). The proportion of carbohydrates was approximately $50 \%$, and that of fat approximately $17 \%$ of the total energy intake. Fiber intake was approximately $14 \mathrm{~g} /$ day during VLCD. The safety and efficacy of the supplement had been considered. During the weight maintenance phase, subjects first gradually replaced the formula meals by conventional meals (reduction of 1 formula meal per week) with increasing energy intake and unchanged proportion of carbohydrates. The protein amount was reduced to $30 \%$ of the total energy uptake, and fat was increased up to $20 \%$. After approximately 5 weeks, subjects exclusively consumed conventional food (up to 1,600 kcal/day) for the rest of weight maintenance period. The carbohydrate proportion remained stable, and the remaining $50 \%$ of the total energy were equally divided into $25 \%$ each for fat and protein. The whole intervention was embedded into a commercial multimodal obesity therapy program for at least 6 months, containing nutritional education, behavioral therapy, and an exercise program in order to ensure weight maintenance after inducing a profound weight loss (Optifast 52) [16]. Inclusion criteria were: between 20 and 66 years of age, Caucasian descent and $\mathrm{BMI}>30 \mathrm{~kg} / \mathrm{m}^{2}$. Baseline characteristics are shown in table 1 . Before inclusion into the study, a written confirmed consent was obtained from each subject. The local ethics committee approved the study (No. D474/11). Six of the 18 subjects suffered from hypertension and 4 from diabetes mellitus type 2, 3 subjects had a substituted hypothyroidism, and 1 each had hyperuricemia, gonarthrosis, psoriasis, obstructive sleep apnea, coxarthrosis, and degenerative spine syndrome. In terms of pharmacotherapy, 4 patients were on diabetic therapy, 4 were taking metformin, and 1 each was on insulin therapy and a therapy with incretin mimetics. Three subjects were taking thyroxine, 2 subjects were taking antidepressant agents, 3 subjects were taking angiotensin II receptor antagonists, 2 were on calcium antagonists, 1 was on AT1 blockers, 3 were on beta blockers, 3 on ACE inhibitors, and 3 on proton pump inhibitors. Four subjects were taking any kind of pain killer. Vitamin or mineral supplements were taken by 4 subjects. One each was taking statins, uricostatics, immune suppressive medication, sedatives, and anticoagulants. One subject received a shortterm antibiotic treatment during the intervention and was therefore excluded from the analysis. Study participants were advised not to change the dosage throughout the study period to achieve stable conditions.

\section{Control Groups}

Two control groups were used in the present study: i) a lean control group (LC, BMI $<25 \mathrm{~kg} / \mathrm{m}^{2}$ ) and ii) an obese control group (OC, BMI $>30 \mathrm{~kg} / \mathrm{m}^{2}$ ). Both control groups did not receive any intervention, and therefore their body weight maintained stable throughout the study period of 6 months. Each group included 13 subjects ( 2 men, 11 women). Inclusion criteria were: between 20 and 66 years of age, Caucasian descent, and $\mathrm{BMI}<25 \mathrm{~kg} / \mathrm{m}^{2}$ ( $\left.\mathrm{LC}\right)$ or $\mathrm{BMI}>30 \mathrm{~kg} / \mathrm{m}^{2}(\mathrm{OC})$. The baseline characteristics of the control groups are shown in table 1 . The controls were embedded in the 'Kiel intervention cohort' as part of the 'Food Chain Plus' study [17]. A written informed consent was obtained from each subject before they were included into this study. 11 of the 26 subjects suffered from hypertension, and 7 showed high blood lipids. Two subjects each had diabetes mellitus, liver disease, migraine, acne, psoriasis, neurodermatitis, and gall stones. One each had cardiac insufficiency, multiple sclerosis, and urticaria. In the past, 3 subjects had cancer and biliary operations, 
Heinsen et al.: Beneficial Effects of a Dietary Weight Loss Intervention on Human Gut

Table 1. Basic characteristics of the study population

\begin{tabular}{|c|c|c|c|c|c|c|c|}
\hline & \multicolumn{3}{|l|}{ Patient groups ${ }^{\mathrm{a}}$} & \multirow[t]{2}{*}{$\mathrm{p}_{\text {total }}$} & \multirow[t]{2}{*}{$p_{1}$} & \multirow[t]{2}{*}{$p_{2}$} & \multirow[t]{2}{*}{$p_{3}$} \\
\hline & VLCD & LC & $\mathrm{OC}$ & & & & \\
\hline Gender, $\%$ female & 83.3 & 84.6 & 84.6 & n.s. & & & \\
\hline Age, years & $\begin{array}{l}47.0 \\
(38.8,54.5)\end{array}$ & $\begin{array}{l}46.0 \\
(37.5,50.5)\end{array}$ & $\begin{array}{l}50.0 \\
(38.5,54.0)\end{array}$ & n.s. & & & \\
\hline Height, m & $\begin{array}{l}1.70 \\
(1.61,1.73)\end{array}$ & $\begin{array}{l}1.68 \\
(1.64,1.77)\end{array}$ & $\begin{array}{l}1.68 \\
(1.62,1.73)\end{array}$ & n.s. & & & \\
\hline Weight, kg & $\begin{array}{l}123.8 \\
(114.1,143.5)\end{array}$ & $\begin{array}{l}64.0 \\
(56.7,71.1)\end{array}$ & $\begin{array}{l}123.5 \\
(107.4,138.1)\end{array}$ & $<0.001$ & $<0.001$ & n.s. & $<0.001$ \\
\hline $\mathrm{BMI}, \mathrm{kg} / \mathrm{m}^{2}$ & $\begin{array}{l}44.5 \\
(38.8,51.5)\end{array}$ & $\begin{array}{l}22.4 \\
(20.7,24.0)\end{array}$ & $\begin{array}{l}42.3 \\
(35.2,47.7)\end{array}$ & $<0.001$ & $<0.001$ & n.s. & $<0.001$ \\
\hline Fasting insulin, $\mu \mathrm{U} / \mathrm{ml}$ & $\begin{array}{l}15.3 \\
(10.4,18.5)\end{array}$ & $\begin{array}{l}8.6 \\
(5.7,10.3)\end{array}$ & $\begin{array}{l}18.2 \\
(12.7,34.8)\end{array}$ & $<0.001$ & 0.001 & n.s. & $<0.001$ \\
\hline Fasting glucose, $\mathrm{mg} / \mathrm{dl}$ & $\begin{array}{l}97.0 \\
(90.5,114.3)\end{array}$ & $\begin{array}{l}87.0 \\
(83.0,110.0)\end{array}$ & $\begin{array}{l}106.0 \\
(89.0,114.6)\end{array}$ & n.s. & & & \\
\hline HOMA-IR index & $3.1(2.6,5.1)$ & $1.9(1.3,2.5)$ & $4.8(3.1,12.5)$ & $<0.001$ & 0.001 & n.s. & $<0.001$ \\
\hline
\end{tabular}

n.s. $=$ Not significant.

$\mathrm{p}_{\text {total }}=\mathrm{p}$ value for overall comparison of the groups determined with the Kruskal-Wallis test with significance level set at $\mathrm{p}<0.05$.

$\mathrm{p}_{1-3}=\mathrm{p}$-values for comparison of the single groups determined with the Mann-Whitney $\mathrm{U}$ test with $\mathrm{p}<$ 0.017 due to the Bonferroni adjustment: $\mathrm{p}_{1}=$ VLCD group versus LC group; $\mathrm{p}_{2}=$ VLCD group versus OC group; $\mathrm{p}_{3}=\mathrm{LC}$ group versus OC group.

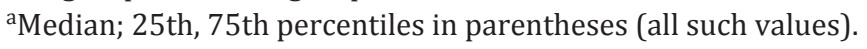

and 2 had venous thrombosis. In terms of pharmacotherapy, 7 patients were taking beta blockers, and 6 subjects were taking thyroxine. Five subjects each were on ACE inhibitors and proton pump inhibitors. Three each were taking statins and glucocorticoids. Two were on diabetic therapy, 1 was taking metformin, and 1 was on insulin therapy. Four were taking antidepressant agents, and 5 were taking diuretics. One each was taking sedatives, uricostatics, chelating agents, calcium antagonists, anticholinergic agents, symphatomimetic drugs, antihistamines, uricosuric agents, and fibrates. Four subjects each were taking vitamin or mineral supplements and angiotensin II receptor antagonists. Two each were on pain killers and anticoagulants. Subjects were advised not to change the dosage throughout the study period to achieve stable conditions.

\section{Data Collection and Biochemical Analyses}

DNA Extraction

DNA was extracted using the QIAamp DNA stool mini kit automated on the QIAcube (Qiagen, Hilden, Germany). Approximately $200 \mathrm{mg}$ stool was transferred to $0.70 \mathrm{~mm}$ Garnet Bead tubes (Dianova, Hamburg, Germany) filled with $1.1 \mathrm{ml}$ ASL lysis buffer. Bead beating was performed using the a SpeedMill PLUS (Analytik Jena, Jena, Germany) for $45 \mathrm{~s}$ at $50 \mathrm{~Hz}$. Samples were then heated to $95^{\circ} \mathrm{C}$ for $5 \mathrm{~min}$ with subsequent continuation of the manufacturer's protocol.

Bacterial 16S rRNA Gene Sequencing and Quality Control

Variable regions V1 and V2 of the 16S rRNA gene were amplified using the primer pair 27F-338R in a dual-barcoding approach according to Caporaso et al. [18]. PCR products were normalized using the 
Heinsen et al.: Beneficial Effects of a Dietary Weight Loss Intervention on Human Gut

Microbiome Diversity and Metabolism Are Not Sustained during Weight Maintenance

SequalPrep Normalization Plate Kit (Thermo Fischer Scientific, Waltham, MA, USA), pooled equimolarily and sequenced on the Illumina MiSeq (Illumina Inc., San Diego, CA, USA). Demultiplexing after sequencing was based on 0 mismatches in the barcode sequences. Forward and reverse reads were merged using the FLASh software, allowing an overlap of the reads between 250 and $300 \mathrm{bp}$ [19]. To eliminate low-quality sequences, the data were filtered by removing sequences with a sequence quality of less than 30 in less than $95 \%$ of the nucleotides. Chimeras were removed with UCHIME [20].

Microbiome Bioinformatic Analysis

Taxonomical classification was carried out using the RDP classifier [21], where classifications with low confidence at genus level $(<0.8)$ are organized in an arbitrary taxon of 'unclassified_[family]'. For each sample, 10,000 sequences were randomly chosen to construct a taxon-by-sample abundance table. To infer functional capabilities from 16S rDNA amplicon sequencing data, reads were matched against the Greengenes OTU database (v13_5) [22], down-sampled to 10,000 reads per sample, and used in the PICRUSt software [23]. To obtain pathway information, the PICRUSt output was used in the HUMAnN software (v0.99) [24].

Anthropometric and Blood Hormone Measurements

Body weight was measured with a Tanita Scale (Body Composition Analyzer; Type BC- 418 MA; Tanita Corporation, Tokyo, Japan), and subjects were interviewed to acquire height. Blood serum was stored immediately at $-80^{\circ} \mathrm{C}$. After determination of fasting insulin by an electro-chemiluminescence immunoassay (ECLIA; Elecsys system; Roche, Basel, Switzerland) and of fasting glucose by an glucose-hexokinase UV test (Hitachi Modular; Roche), the Homeostasis Model Assessment (HOMA) index was calculated as glucose $(\mathrm{mg} / \mathrm{dl}) \times \operatorname{insulin}(\mu \mathrm{U} / \mathrm{ml}) / 405$.

\section{Statistical Analysis}

Statistical analyses were carried out with SPSS, version 22.0 for windows (SPSS, Chicago, IL, USA). Graphic data analysis was performed with GraphPad Prism, version 5.0 (GraphPad Software, San Diego, CA, USA). A test to determine deviations from normal distribution would have had low statistical power due to the small sample size. Therefore, data are shown as median (IQ) due to Tukey, and exclusively statistical tests for non-parametric data were used. Categorical variables are presented as percentages. The overall comparison between the 3 groups (VLDC, LC, OC) was performed by the Kruskal-Wallis test. When significant, the pairwise comparison was carried out by the Mann-Whitney U test. The chi square test was used to determine differences in categorical variables. To identify statistically significant differences between time points, the Friedman test and Wilcoxon signed-rank test were applied. The significance level was set at $\mathrm{p}<$ 0.05. If multiple comparisons were made, the significance level was set at $p<0.017$, due to the Bonferroni adjustment.

Statistical analyses of the microbiome data were performed in R, using the 'vegan' package [25]. A permutational multivariate analysis of variance (PERMANOVA) was performed by applying the 'adonis' function to the Bray-Curtis dissimilarity [26]. For the analysis of changes between different time points, individuals with samples available for all time points were included. Unconstrained principal component analysis (PCoA) was carried out using R/vegdist ( $\mathrm{n}=13$ for the OC and LC groups at time points 0 and 6; $n=18,11$ and 16 for the VLCD group at time points 0,3 and 6, respectively), and the 'capscale' function was used for the constrained analysis of principal coordinates $(n=8)[27,28]$. The indicator species analysis within the VLCD group was performed using the multi-level pattern analysis method ('multipatt'), calculating the indicator value (IndVal) metric as implemented in the 'indicspecies' package for R [29]. Unfortunately, some patients did not provide the study center with stool samples for every time point. Under these circumstances, a patient was only included into the statistical microbiome analysis if stool samples were available for all 3 time points $(0,3,6$ months $)(n=8)$.

\section{Results}

Body Weight, BMI, and Insulin Sensitivity during Weight Loss and Weight Maintenance The present study was conducted to address 2 major questions: i) Does a 3-month formula-based VLCD (approximately $800 \mathrm{kcal} /$ day) obesity program significantly alter the gut microbiota diversity and/or metabolism? ii) Are these potentially beneficial changes in 
Table 2. Changes in bioclinical parameters during the study (mean \pm SEM)

\begin{tabular}{llll}
\hline & Baseline & 3 months & 6 months \\
\hline Systolic blood pressure, mm Hg & $144 \pm 5$ & $133 \pm 5$ & $127 \pm 3$ \\
\hline Diastolic blood pressure, mm Hg & $89 \pm 2$ & $85 \pm 2$ & $79 \pm 4$ \\
\hline Leukocytes, $10^{9} / \mathrm{l}$ & $6.5 \pm 0.3$ & $6.3 \pm 0.4$ & $6.1 \pm 0.3$ \\
\hline Hemoglobin, g/l & $14.1 \pm 0.2$ & $14.1 \pm 0.3$ & $13.3 \pm 0.9$ \\
\hline Creatinine, mg/dl & $0.9 \pm 0.1$ & $0.8 \pm 0.1$ & $0.8 \pm 0.1$ \\
\hline Uric acid, mg/dl & $6.3 \pm 0.4$ & $5.4 \pm 0.4$ & $4.3 \pm 0.6$ \\
\hline ALT, U/l & $28.4 \pm 2.5$ & $28.8 \pm 4.1$ & $31.9 \pm 12.7$ \\
\hline yGT, U/l & $50.3 \pm 13.3$ & $51.5 \pm 16.3$ & $67.1 \pm 22.1$ \\
\hline Triglycerides, mg/dl & $124.4 \pm 14.6$ & $108.7 \pm 15.8$ & $110.0 \pm 17.6$ \\
\hline Glucose, mg/dl & $99.0 \pm 7.6$ & $91.3 \pm 1.7$ & $97.6 \pm 8.6$ \\
\hline Sodium, mmol/l & $139.3 \pm 0.6$ & $140.1 \pm 0.4$ & $130.8 \pm 8.2$ \\
\hline Potassium, mmol/l & $4.1 \pm 0.1$ & $4.1 \pm 0.1$ & $4.0 \pm 0.3$ \\
\hline
\end{tabular}

$\mathrm{ALT}=$ Alanine-aminotransferase, $\mathrm{yGT}=\gamma$-glutamyltransferase

the gut microbiota sustained during an additional 3-month weight maintenance phase? It is important to note that until today, the composition of the 'normal' and 'healthy' gut microbiome is still unclear. In contrast to a previous report examining a dietary weight loss intervention [30], we therefore included 2 distinct control groups in addition to the VLCD intervention group in our study: the first control group (OC, BMI $>30 \mathrm{~kg} / \mathrm{m}^{2}$ ) consisted of obese individuals having the same body weight as the intervention group, but receiving no VLCD. The second control group ( $\mathrm{LC}, \mathrm{BMI}<25 \mathrm{~kg} / \mathrm{m}^{2}$ ) consisted of lean, metabolically healthy human subjects who did not receive any type of dietary intervention either. While the body weight of the intervention group decreased significantly during the study period, there was no major weight change in either control group. The basic characteristics of the different study groups are shown in table 1.

Three months of a formula-based VLCD intervention resulted in a profound weight loss from $123.75 \mathrm{~kg}$ (IQ range $114.08-143.45 \mathrm{~kg}$ ) to $102.30 \mathrm{~kg}(87.73-120.25 \mathrm{~kg})(\mathrm{p}<0.001)$. We measured fasting insulin and fasting glucose levels and calculated the HOMA index. This parameter showed a significant reduction due to the dietary intervention from 3.09 (2.615.14) to $1.73(1.03-3.7)(p<0.01)$, indicating an improvement of insulin sensitivity. During the following 3 months of weight maintenance, the body weight could be stabilized and accounted to $99.35 \mathrm{~kg}(87.73-120.05 \mathrm{~kg})$ at the end of the study period. The HOMA index settled at 2.11 (1.51-3.87) during weight maintenance. The bioclinical parameters during intervention are given in table 2 . Since the control groups did not undergo an intervention, no significant changes of bioclinical parameters could be observed. 
Fig. 1. CAP of the dietary intervention group explaining variance in the microbiota. Baseline $(0$ months $)=0$, VLCD dietary intervention ( 3 months) $=3$, weight maintenance period $(6$ months $)=$ 6.

Heinsen et al.: Beneficial Effects of a Dietary Weight Loss Intervention on Human Gut

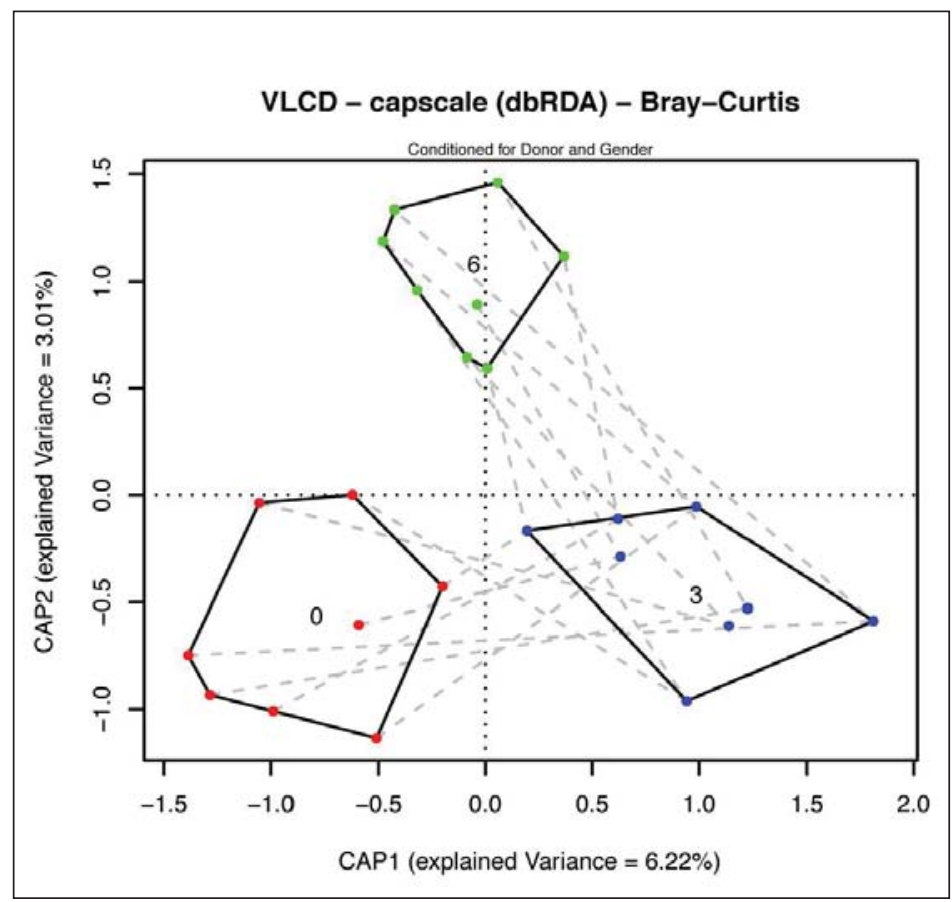

Gut Microbiome Diversity during Weight Loss and Weight Maintenance

On phylum or genus level, a significant difference $(\mathrm{p}=0.047)$ between the LC and VLDC group could be observed at baseline, when calculating the Firmicutes/Bacteroidetes ratio, while no significant difference was found between the OC and VLDC group. We next performed a PERMANOVA analysis, using the Bray-Curtis dissimilarity, which is a beta-diversity measure that reflects inter-individual variability, in order to examine differences within the groups during the time period of the study. For LC, this analysis did not show a significant change in variance over time $\left(\mathrm{R}^{2}=0.00788, \mathrm{p}=0.8622\right)$. In addition, for $\mathrm{OC}$, no significant change occurred over time either $\left(\mathrm{R}^{2}=0.02731, \mathrm{p}=0.3312\right)$. In contrast, the VLCD intervention group exhibited a significant correlation between the time points and the Bray-Curtis dissimilarity $\left(R^{2}=0.11074, p=0.009799\right)$. A subsequent analysis revealed the significant difference in diversity in the VLCD group between baseline ( 0 months) and the end of the nutritional intervention ( 3 months; $\mathrm{R}^{2}=0.076763, \mathrm{p}=0.005299$ ), while no significant differences between the end of the nutritional intervention ( 3 months) and the end of the weight maintenance phase ( 6 months; $\mathrm{R}^{2}=0.04317, \mathrm{p}=0.08309$ ) or between baseline ( 0 months) and the end of the weight maintenance phase were detected ( 6 months; $\left.R^{2}=0.02045, p=0.2679\right)$. In a constrained analysis of principal coordinates (CAP), the first coordinate explained $6.22 \%$ (p $=0.048$ ) of the variance in the microbiota due to the VLCD intervention, whereas the second coordinate explained only $3.01 \%$ and was not significant $(p=0.9422)$ (fig. 1$)$. It has to be pointed out that neither at baseline ( 0 months) nor at the end of the study period ( 6 months) significant differences between the 3 groups could be observed on phylum or genus level, indicating that the significant changes induced by the VLCD intervention completely disappeared within the weight maintenance phase (fig. 2). Taken together, significant changes in the gut microbiome diversity were only detected in the VLCD group directly after the VLCD intervention. During the following weight maintenance period, these changes regressed towards the measurements at baseline. 


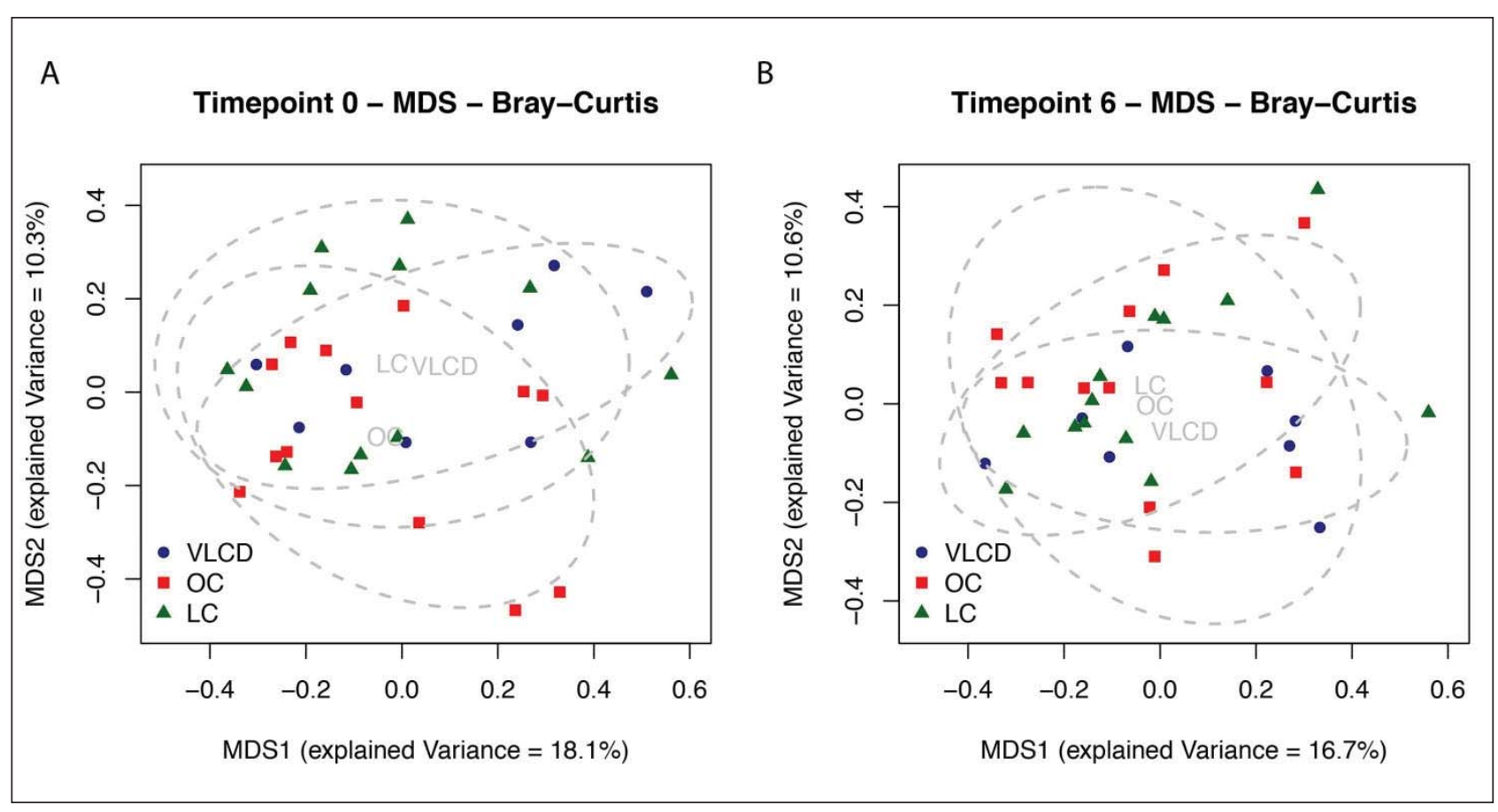

Fig. 2. Unconstrained PCoA of the intervention group and both control groups. A: at the beginning of the study period ( 0 months). B: at the end of the study period ( 6 months).

Defined Species of the Gut Microbiome during Weight Loss and Weight Maintenance

Having identified significant changes in gut microbiome diversity due to the VLCD intervention, we next used IndVal in order to identify indicator species accounting for the diversity change within the intervention group at 3 months of the study period. Only genera with an abundance of at least $1 \%$ in one of the samples were included in this analysis. Interestingly, this analysis identified a significant association with the genus Acinetobacter (IndVal $=0.998$, $\mathrm{p}=0.006$, after Benjamini-Hochberg adjustment). This statistically significant association was confirmed by a Wilcoxon rank-sum test $(\mathrm{p}=0.042)$. Figure 3 shows the abundance of Acinetobacter over time due to VLCD intervention.

\section{Bacterial Metabolic Changes during Weight Loss and Weight Maintenance}

From a pathophysiological point of view, not the presence or absence of a certain microorganism might be of importance, but the metabolic capacity of the microbiota because it might be possible that 2 different bacteria share the same major metabolic capacity. In that case, the absence of one organism might be compensated by the presence of the other, resulting in similar overall metabolic activity of the gut microbiome. For that reason, we also performed a PICRUSt/HUMAnN analysis, using the KEGG database for metabolic pathways. Amongst other things, this analysis revealed changes in the riboflavin pathway and the chlorocyclohexane and chlorobenzene degradation pathways at 3 months of the study period. These changes were nominal statistically significant $\left(\mathrm{p}_{\text {nom }}=0.0078\right.$ and $\left.\mathrm{p}_{\text {nom }}=0.039\right)$ but not robust to multiple testing. During the weight maintenance phase, these metabolic changes diminished, showing a time course pattern comparable to the diversity measures (fig. 4). 
Fig. 3. Abundance of indicator genus Acinetobacter over time in the VLCD group. Increase of Acinetobacter due to VLCD intervention is statistically significant. Baseline $(0$ months $)=0$, VLCD dietary intervention (3 months) $=3$, weight maintenance period $(6$ months) $=6$.

Fig. 4. Relative abundance of the riboflavin pathway during the dietary intervention. Nominal significant differences between time points. Baseline $(0$ months $)=0$, VLCD dietary intervention (3 months) $=3$, weight maintenance period $(6$ months $)=6$.
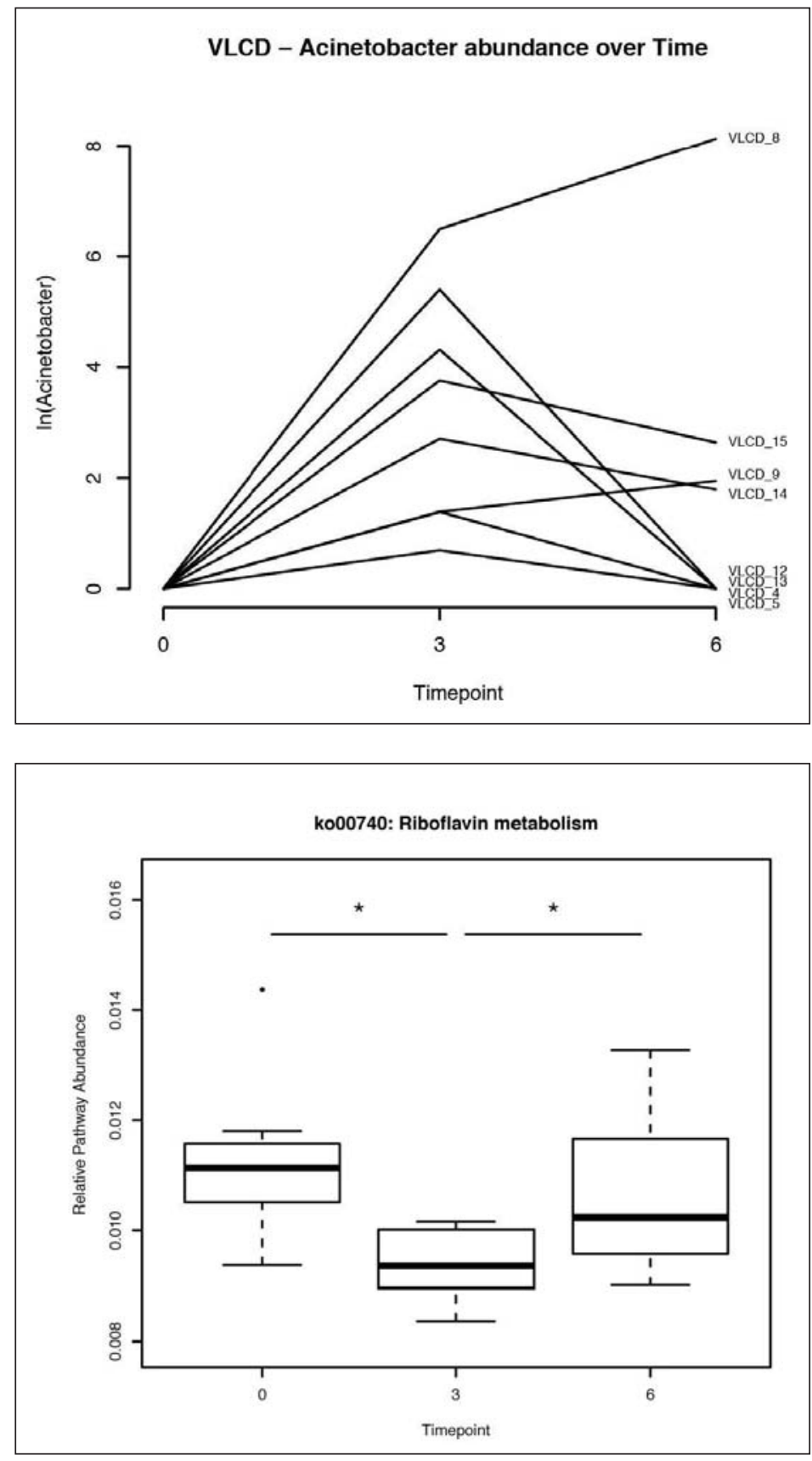

\section{Discussion}

While several mouse studies exhibited a significant association of the gut microbiome with metabolic abnormalities like obesity and insulin resistance [7], data in humans are still conflicting [9-11]. In the present study we used a VLCD-based obesity program in obese subjects for two reasons: i) This kind of diet is completely standardized and fully balanced with micro- and macronutrients, excluding inter-individual differences in food consumption, and ii) it results in a profound weight loss in a short period of time, inducing an enormous shift in the human metabolic phenotype [31, 32]. 
In agreement with several animal models [7] and with some of the human studies [9], we identified an altered Firmicutes/Bacteroidetes ratio between the LC and VLCD group, whereas no difference was found between both obese groups. While many studies compare microbiome data at a single time point between larger cohorts of various BMI, we aimed to study a smaller cohort in more depth (diversity and bacterial metabolism) and in a longitudinal approach, in order to take into account intra-individual changes of the microbiome over time. Regarding the intra-individual microbiome, our analysis revealed that there were no significant changes in the microbiome diversity of both LC and OC within 6 months, indicating that, regardless of the individual BMI, a stable lifestyle results in a more or less stable composition of the gut microbiome.

A VLCD intervention of obese humans over a time period of 3 months resulted in i) a significant change in gut microbiome diversity, ii) a significant increase of the abundance of an indicator species, and iii) a significant alteration of specific bacterial metabolic pathways. Although no significant increase in body weight occurred within the weight maintenance period, all the changes in diversity and bacterial metabolism mentioned above diminished. This finding suggests that nutritional components exert a stronger effect on the human gut microbiome than changes in body weight or insulin sensitivity. Weight gain is often observed in the long term after VLCD-based obesity programs [16] which might be due to many independent factors (e.g., dietary composition, age, and sex). The present data suggest that a remission of favorable changes in the gut microbiome induced by a diet might also be partly responsible.

Moreover, previous studies indicate that not only the caloric intake but also the macronutrient composition of the diet influences microbiota shifts. The VLCD used in this study consisted of a relatively high amount of protein and a relatively low amount of fat. Additionally, the fiber intake during the VLCD might be higher than the common fiber intake during usual diets of obese subjects. During the weight maintenance period, the proportion of protein was reduced and the proportion of fat was increased. A low fat/high fiber diet, as it was performed during VLCD, was shown to change microbial composition in a controlled feeding study [33]. The impact of a high-fiber diet on microbiome was also shown by DeFillipo et al. [34]. Furthermore, a high-protein diet was shown to have induced a change in microbial composition in 19 healthy obese subjects [35].

In 2010, a study was published on the effect of bariatric surgery (Roux-Y gastric bypass; RYGB) on human gut microbiota [36]. The mean body weight of the 30 obese subjects in that study accounted to $126 \pm 4.2 \mathrm{~kg}$ and decreased approximately by $20 \mathrm{~kg}$ within the first 3 months. After that, the body weight still decreased, although the reduction was less when compared to the first 3 months after the surgical procedure. Thus, both the baseline body weight and the time course of the weight reduction are comparable to that of our study. In agreement to our findings, the authors found significant changes in the gut microbiome within the first 3 months after RYGB. In contrast to our non-surgical approach, changes in the gut microbiome remained stable between the 3rd and 6th month after the gastric bypass, however (e.g., Bacteroidetes levels) [36]. This is particularly interesting since the long-term effects on body weight are more convincing after bariatric surgery when compared to non-surgical obesity therapies [37]. Thus, the differences in terms of stabilization of gut microbiota changes within the weight maintenance phase between the nonsurgical approach in our study and the effects of RYGB might at least in part account for the better long-term effects of surgical versus nonsurgical obesity therapies [37].

In addition to the analysis of microbiome diversity, we further performed a PICRUSt/ HUMAnN analysis, employing the KEGG database to examine bacterial metabolic pathways. In this analysis, two metabolic pathways were identified with a significant alteration in the VLCD phase followed by a remission in the weight maintenance, mirroring the diversity 
Heinsen et al.: Beneficial Effects of a Dietary Weight Loss Intervention on Human Gut

changes. The highest level of significance was found for the riboflavin (vitamin B2) pathway, which was decreased by the VLCD (fig. $4, \mathrm{p}_{\text {nom }}=0.0078$ ). This is of interest since humans are not capable of producing sufficient amounts of B vitamins themselves, and B vitamin deficiency is therefore common [38]. Furthermore, a low riboflavin intake has been shown to be inversely associated with greater adiposity [39]. The B vitamin production by the gut microbiome plays an important role in supplying humans with these essential nutrients under a regular diet [40]. Since the VLCD in our study was formula-based and thereby fully balanced in micro- and macronutrients, it can be speculated that the gut microbiome was able to sense the better supply of riboflavin by the VLCD and possibly adopted its metabolic activity (e.g., decrease in microbial riboflavin biosynthesis). However, serum concentrations were not measured in subjects of this study, and further investigations should be performed in future studies to prove this hypothesis.

It also should be mentioned that our study has limitations: i) The number of subjects is limited, ii) several obese subjects were suffering from additional diseases and were taking medication, and iii) the number of male subjects was limited in our cohorts. However, we feel that the results of the present study indicate that a VLCD-based obesity program is able to alter both gut microbiome diversity and metabolism in obese humans, but that these changes are not sustained during weight maintenance. This suggests that targeting the microbiome (e.g., by probiotics or fecal transplantation) in addition to restricting the caloric intake might be a promising future approach for maintaining a significant weight loss after a dietary intervention.

\section{Acknowledgements}

We thank all the patients who participated in the study. This work was supported by the German Federal Ministry of Education and Research (BMBF) within the framework of the e:Med research and funding program (grant \# 01ZX1306A) and the DFG Cluster of Excellence 'Inflammation at Interfaces'.

\section{Disclosure Statement}

Matthias Laudes, Dominik Schulte, and Ute Settgast are providers of the Optifast 52 Obesity Therapy Program at the University Hospital in Kiel. No other authors declare a conflict of interest.

\section{References}

1 WHO: Obesity and Overweight. Fact Sheet. http://www.who.int/mediacentre/factsheets/fs311/en/ (last accessed October 19, 2016).

2 Sommer F, Bäckhed F: The gut microbiota - masters of host development and physiology. Nat Rev Microbiol 2013;11:227-238.

3 Bäckhed F, Ding H, Wang T, Hooper LV, Koh GY, Nagy A, Semenkovich CF, Gordon JI: The gut microbiota as an environmental factor that regulates fat storage. Proc Natl Acad Sci U S A 2004;101:15718-15723.

4 Turnbaugh PJ, Ley RE, Mahowald MA, Magrini V, Mardis ER, Gordon JI: An obesity-associated gut microbiome with increased capacity for energy harvest. Nature 2006;444:1027-1031.

5 Caesar R, Reigstad CS, Bäckhed HK, Reinhardt C, Ketonen M, Lundén GO, Cani PD, Bäckhed F: Gut-derived lipopolysaccharide augments adipose macrophage accumulation but is not essential for impaired glucose or insulin tolerance in mice. Gut 2012;61:1701-1707.

6 Ley RE, Backhed F, Turnbaugh P, Lozupone CA, Knight RD, Gordon JI: Obesity alters gut microbial ecology. Proc Natl Acad Sci U S A 2005;102:11070-11075.

7 Turnbaugh PJ, Bäckhed F, Fulton L, Gordon JI: Diet-induced obesity is linked to marked but reversible alterations in the mouse distal gut microbiome. Cell Host Microbe 2008;3:213-223.

8 Ley RE, Turnbaugh PJ, Klein S, Gordon JI: Microbial ecology: human gut microbes associated with obesity. Nature 2006;444:1022-1023. 
Heinsen et al.: Beneficial Effects of a Dietary Weight Loss Intervention on Human Gut

9 Jumpertz R, Le DS, Turnbaugh PJ, Trinidad C, Bogardus C, Gordon JI, Krakoff J: Energy-balance studies reveal associations between gut microbes, caloric load, and nutrient absorption in humans. Am J Clin Nutr 2011;94: $58-65$.

10 Million M, Maraninchi M, Henry M, Armougom F, Richet H, Carrieri P, Valero R, Raccah D, Vialettes B, Raoult D: Obesity-associated gut microbiota is enriched in Lactobacillus reuteri and depleted in Bifidobacterium animalis and Methanobrevibacter smithii. Int J Obes (Lond) 2012;36:817-825.

11 Duncan SH, Lobley GE, Holtrop G, Ince J, Johnstone AM, Louis P, Flint HJ: Human colonic microbiota associated with diet, obesity and weight loss. Int J Obes (Lond) 2008;32:1720-1724.

12 Kong LC, Holmes BA, Cotillard A, Habi-Rachedi F, Brazeilles R, Gougis S, Gausseres N, Cani PD, Fellahi S, Bastard JP, Kennedy SP, Dore J, Ehrlich SD, Zucker JD, Rizkalla SW, Clement K: Dietary patterns differently associate with inflammation and gut microbiota in overweight and obese subjects. PLoS One 2014; 9:e109434.

13 Vrieze A, Van Nood E, Holleman F, Salojärvi J, Kootte RS, Bartelsman JF, Dallinga-Thie GM, Ackermans MT, Serlie MJ, Oozeer R, Derrien M, Druesne A, Van Hylckama Vlieg JE, Bloks VW, Groen AK, Heilig HG, Zoetendal EG, Stroes ES, de Vos WM, Hoekstra JB, Nieuwdorp M: Transfer of intestinal microbiota from lean donors increases insulin sensitivity in individuals with metabolic syndrome. Gastroenterology 2012;143:913-916.e917.

14 Le Chatelier E, Nielsen T, Qin J, Prifti E, Hildebrand F, Falony G, Almeida M, Arumugam M, Batto JM, Kennedy S, Leonard P, Li J, Burgdorf K, Grarup N, Jorgensen T, Brandslund I, Nielsen HB, Juncker AS, Bertalan M, Levenez F, Pons N, Rasmussen S, Sunagawa S, Tap J, Tims S, Zoetendal EG, Brunak S, Clement K, Dore J, Kleerebezem M, Kristiansen K, Renault P, Sicheritz-Ponten T, de Vos WM, Zucker JD, Raes J, Hansen T, Bork P, Wang J, Ehrlich SD, Pedersen O: Richness of human gut microbiome correlates with metabolic markers. Nature 2013;500: 541-546.

15 Cotillard A, Kennedy SP, Kong LC, Prifti E, Pons N, Le Chatelier E, Almeida M, Quinquis B, Levenez F, Galleron N, Gougis S, Rizkalla S, Batto JM, Renault P, Dore J, Zucker JD, Clement K, Ehrlich SD: Dietary intervention impact on gut microbial gene richness. Nature 2013;500:585-588.

16 Bischoff SC, Damms-Machado A, Betz C, Herpertz S, Legenbauer T, Löw T, Wechsler JG, Bischoff G, Austel A, Ellrott T: Multicenter evaluation of an interdisciplinary 52 -week weight loss program for obesity with regard to body weight, comorbidities and quality of life - a prospective study. Int J Obes (Lond) 2012;36:614-624.

17 Müller N, Schulte DM, Türk K, Freitag-Wolf S, Hampe J, Zeuner R, Schroder JO, Gouni-Berthold I, Berthold HK, Krone W, Rose-John S, Schreiber S, Laudes M: IL-6 blockade by monoclonal antibodies inhibits apolipoprotein (a) expression and lipoprotein (a) synthesis in humans. J Lipid Res 2015;56:1034-1042.

18 Caporaso JG, Lauber CL, Walters WA, Berg-Lyons D, Huntley J, Fierer N, Owens SM, Betley J, Fraser L, Bauer M, Gormley N, Gilbert JA, Smith G, Knight R: Ultra-high-throughput microbial community analysis on the Illumina HiSeq and MiSeq platforms. ISME J 2012;6:1621-1624.

19 Magoc T, Salzberg SL: FLASH: fast length adjustment of short reads to improve genome assemblies. Bioinformatics 2011;27:2957-2963.

20 Edgar RC, Haas BJ, Clemente JC, Quince C, Knight R: UCHIME improves sensitivity and speed of chimera detection. Bioinformatics 2011;27:2194-2200.

21 Wang Q, Garrity GM, Tiedje JM, Cole JR: Naive Bayesian classifier for rapid assignment of rRNA sequences into the new bacterial taxonomy. Appl Environ Microbiol 2007;73:5261-5267.

22 DeSantis TZ, Hugenholtz P, Larsen N, Rojas M, Brodie EL, Keller K, Huber T, Dalevi D, Hu P, Andersen GL: Greengenes, a chimera-checked 16S rRNA gene database and workbench compatible with ARB. Appl Environ Microbiol 2006;72:5069-5072.

23 Langille MG, Zaneveld J, Caporaso JG, McDonald D, Knights D, Reyes JA, Clemente JC, Burkepile DE, Vega Thurber RL, Knight R, Beiko RG, Huttenhower C: Predictive functional profiling of microbial communities using 16S rRNA marker gene sequences. Nat Biotechnol 2013;31:814-821.

24 Abubucker S, Segata N, Goll J, Schubert AM, Izard J, Cantarel BL, Rodriguez-Mueller B, Zucker J, Thiagarajan M, Henrissat B, White O, Kelley ST, Methe B, Schloss PD, Gevers D, Mitreva M, Huttenhower C: Metabolic reconstruction for metagenomic data and its application to the human microbiome. PLoS Comput Biol 2012; 8:e1002358.

25 Dixon P: VEGAN, a package of R functions for community ecology. J Veg Sci 2003;14:927-930.

26 Anderson MJ: A new method for non-parametric multivariate analysis of variance. Austr Ecol 2001;26:32-46.

27 Anderson MJ, Willis TJ: Canonical analysis of principal coordinates: a useful method of constrained ordination for ecology. Ecology 2003;84:511-525.

28 Legendre P, Anderson MJ: Distance-based redundancy analysis: testing multispecies responses in multifactorial ecological experiments. Ecol Monogr 1999;69:1-24.

29 De Cáceres M, Legendre P: Associations between species and groups of sites: indices and statistical inference. Ecology 2009;90:3566-3574.

30 Simões CD, Maukonen J, Scott KP, Virtanen KA, Pietilainen KH, Saarela M: Impact of a very low-energy diet on the fecal microbiota of obese individuals. Eur J Nutr 2014;53:1421-1429.

31 Hagen I, Schulte DM, Müller N, Martinsen J, Türk K, Hedderich J, Schreiber S, Laudes M: Soluble receptor for advanced glycation end products as a potential biomarker to predict weight loss and improvement of insulin sensitivity by a very low calorie diet of obese human subjects. Cytokine 2015;73:265-269.

32 Oberhauser F, Schulte DM, Faust M, Gudelhofer H, Hahn M, Müller N, Neumann K, Krone W, Laudes M: Weight loss due to a very low calorie diet differentially affects insulin sensitivity and interleukin-6 serum levels in nondiabetic obese human subjects. Horm Metab Res 2012;44:465-470. 
Heinsen et al.: Beneficial Effects of a Dietary Weight Loss Intervention on Human Gut

33 Wu GD, Chen J, Hoffmann C, Bittinger K, Chen YY, Keilbaugh SA, Bewtra M, Knights D, Walters WA, Knight R, Sinha R, Gilroy E, Gupta K, Baldassano R, Nessel L, Li H, Bushman FD, Lewis JD: Linking long-term dietary patterns with gut microbial enterotypes. Science 2011;334:105-108.

34 De Filippo C, Cavalieri D, Di Paola M, Ramazzotti M, Poullet JB, Massart S, Collini S, Pieraccini G, Lionetti P: Impact of diet in shaping gut microbiota revealed by a comparative study in children from Europe and rural Africa. Proc Natl Acad Sci U S A 2010;107:14691-14696.

35 Duncan SH, Belenguer A, Holtrop G, Johnstone AM, Flint HJ, Lobley GE: Reduced dietary intake of carbohydrates by obese subjects results in decreased concentrations of butyrate and butyrate-producing bacteria in feces. Appl Environ Microbiol 2007;73:1073-1078.

36 Furet JP, Kong LC, Tap J, Poitou C, Basdevant A, Bouillot JL, Mariat D, Corthier G, Dore J, Henegar C, Rizkalla S, Clement K: Differential adaptation of human gut microbiota to bariatric surgery-induced weight loss: links with metabolic and low-grade inflammation markers. Diabetes 2010;59:3049-3057.

37 Schauer PR, Bhatt DL, Kirwan JP, Wolski K, Brethauer SA, Navaneethan SD, Aminian A, Pothier CE, Kim ES, Nissen SE, Kashyap SR: Bariatric surgery versus intensive medical therapy for diabetes - 3-year outcomes. $N$ Engl J Med 2014;370:2002-2013.

38 Rucker RB, Zempleni J, Suttie JW, Mccormick DB (eds): Handbook of Vitamins, ed. 4, Boca Raton, CRC Press, 2007.

39 Gunanti IR, Marks GC, Al-Mamun A, Long KZ: Low serum vitamin B-12 and folate concentrations and low thiamin and riboflavin intakes are inversely associated with greater adiposity in Mexican American children. J Nutr 2014;144:2027-2033.

40 Hill MJ: Intestinal flora and endogenous vitamin synthesis. Eur J Cancer Prev 1997;6(suppl 1):43-45. 